\title{
Differential expression of aquaporin 5 and aquaporin 3 in squamous cell carcinoma and adenoid cystic carcinoma
}

\author{
SHUNSUKE ISHIMOTO ${ }^{1,2}$, KOICHIRO WADA $^{1}$, YU USAMI $^{3}$, NORIAKI TANAKA ${ }^{2}$, \\ TOMONAO AIKAWA ${ }^{2}$, MASAYA OKURA ${ }^{2}$, ATSUSHI NAKAJIMA ${ }^{4}$, \\ MIKIHIKO KOGO ${ }^{2}$ and YOSHINORI KAMISAKI ${ }^{1}$ \\ ${ }^{1}$ Department of Pharmacology and ${ }^{2}$ The First Department of Oral and Maxillofacial Surgery, Graduate School of Dentistry, \\ Osaka University; ${ }^{3}$ Clinical Laboratory, Osaka University Dental Hospital, Suita, Osaka 565-0871; \\ ${ }^{4}$ Department of Gastroenterology, Yokohama City University School of Medicine, \\ Yokohama 236-0004, Japan
}

Received December 28, 2011; Accepted March 9, 2012

DOI: $10.3892 /$ ijo.2012.1445

\begin{abstract}
Aquaporins (AQPs) are a membrane protein family involved in the selective transport of water across cell membranes. Recent studies have reported the expression of AQP5 in several tumor types such as gastric, pulmonary, ovarian, pancreatic and colorectal cancer. We have previously reported the expression on tumor cells and the important role of AQP3 on cell growth in tongue cancer. However, little is known about the expression and precise role of AQP5 on squamous cell carcinoma (SCC) of the tongue. We investigated the expression of AQP5 and AQP3 in human oral SCC and adenoid cystic carcinoma (ACC). Overexpression of both AQP5 and AQP3 were immunohistochemically observed on tumor cells in SCC, whereas ACC cells were faintly stained with those antibodies against AQPs. Treatment with pan-AQP inhibitor or specific AQP5-siRNA showed inhibition of cell growth in SCC cell lines via the inhibition of integrins and the mitogen-activated protein kinase pathway. AQPs play important roles in cell growth in SCC rather than ACC.
\end{abstract}

\section{Introduction}

Human squamous cell carcinoma (SCC) is a major neoplasm in oral cavity and the incidence is increasing (1-3). The optimal treatment or therapy for early carcinoma is a surgical operation. However, overall survival remains largely unchanged (1-3). In addition, the decrease in quality of life (QOL) after the wide

Correspondence to: Dr Shunsuke Ishimoto, Department of Pharmacology, Graduate School of Dentistry, Osaka University, 1-8 Yamadaoka, Suita, Osaka 565-0871, Japan

E-mail: ishimoto@dent.osaka-u.ac.jp

Key words: squamous cell carcinoma, adenoid cystic carcinoma, cellular proliferation, aquaporin, integrin, mitogen activated protein kinase excision of tongue is also important issue for patients. Therefore, different therapies for the inhibition of tumor cell growth are required.

The aquaporin (AQP) family is a membrane protein involved in the selective transport of water across cell membranes (4). Several subsets of AQP also transport small molecules such as glycerol and urea (5). Among them, AQP5, transports only water, is known to be expressed in various organs such as lung and saliva gland, and AQP3, transports not only water but also glycerol and urea, is known to be expressed in kidney, skin, lung and gastrointestinal tracts (6-11). It has been reported that several tumor cell types also express AQPs in humans and they may be involved in cell proliferation, migration and metastasis (12). Recent studies have also reported the expression of AQP5 in tumors such as gastric, pulmonary, ovarian, pancreatic and colorectal cancer (13-16). However, little is known about the expression and exact role of AQP5 on SCC of the tongue.

Recently, we have reported the expression on tumor cells and important role on cell growth of AQP3 in tongue cancer (17). Therefore, we hypothesised that other AQPs, such as AQP5, might express and play an important role on the growth of SCCs. In the present study, we examined whether AQP5 is expressed in various oral cancers, such as SCC, adenoid cystic carcinoma (ACC). In addition, we investigated the co-expressions of AQP5 and AQP3. Our results imply a potentially important and novel role for AQP5 and AQP3 functions on various types of oral cancers and the inhibition of AQPs in SCCs might be a useful therapy.

\section{Materials and methods}

Tissue samples. The clinical studies were approved by the Ethics committee of Osaka University Dental Hospital. Twenty four samples of SCC located in the tongue, and 13 samples of ACC located in the minor or major salivary gland were obtained by surgical resection of tissue specimens at Osaka University Dental Hospital after informed consent and agreement were obtained. The patients, who received no preoperative therapy including chemotherapy and irradiation therapy, were studied 
(Table I and II). The age range was $30-92$ years (average: $59.5 \pm 16.0$ years, mean $\pm \mathrm{SD}$ ) in SCC patients, 29-79 years (average: $57.1 \pm 16.5$ years, mean $\pm \mathrm{SD}$ ) in ACC patients, respectively.

Chemicals and antibodies. $\mathrm{CuSO}_{4}$ was from Wako Pure Chemical Industries Ltd (Osaka, Japan). Anti-AQP5, AQP3, integrin $\alpha 5$ and integrin $\beta 1$ polyclonal antibody were from Santa Cruz Biotechnology (Santa Cruz, CA, USA). Antibodies against focal adhesion kinase (FAK) and p44/42MAPK (Erk1/2) are from Cell Signalling Technologies (Beverly, MA, USA).

Immunohistochemical staining of $A Q P 5$ and $A Q P 3$. The expression of AQP5 or AQP3 in tissues was detected by anti-AQP5 or AQP3 antibody using standard immunohistochemical techniques on formalin-fixed and paraffin-embedded continuous sections. Briefly, incubation with anti-AQP5 or AQP3 polyclonal antibody was performed at $4{ }^{\circ} \mathrm{C}$ for $16 \mathrm{~h}$, then the sections were washed. After the application with secondary antibody, the Vectastain ABC kit (Vector Laboratories, Burlingame, CA, USA) was used with a 3,3'-diaminobenzidine (DAB) substrate kit, according to the manufacturer's instructions. The staining endpoint was determined when the standard tissue sections were repeatedly stained the intensity as described previously $(17,18)$.

The intensity of the immunohistochemical staining with anti-AQP5 or AQP3 antibody was evaluated by scoring the staining reaction in four groups: -, below $10 \%$ of the expression; ,$+ 10-20 \%$ of the expression;,$++ 20-50 \%$ of the expression; and +++ , over $50 \%$ cytoplasmic staining intensity, respectively $(17,18)$. To confirm the reproducibility, the re-evaluation concerning the immunohistochemical staining for the AQP5 and AQP3 was conducted by oral surgeon and pathologist who were unaware of the original assessment. Non-tumor areas were selected as the comparatively normal areas are separated far away from the tumor areas and confirmed by the pathologist.

Cell culture. We used human oral SCC cell lines (SAS, SCCKN and Ca9-22) and fibroblasts. SAS and SCCKN were established tongue SCC, and Ca9-22 was established gingival SCC $(19,20)$. SAS, SCCKN and fibroblasts were maintained in DMEM containing 10\% fetal bovine serum (FBS), and Ca9-22 was maintained in DMEM containing 10\% FBS and $0.6 \%$ glutamine at $37^{\circ} \mathrm{C}$ under $0.5 \% \mathrm{CO}_{2}$. For the cell growth experiment, cells were trypsinized and replated onto culture dishes (17-21).

Fluorescent microscopy. Analysis of F-actin structure by fluorescent microscopy was carried out according to a method described by the manufacturer. Briefly, SCC cells were cultured on a Laboratory-Tek II chamber slide. Cells were treated with pan-AQP inhibitor, $\mathrm{CuSO}_{4}(100 \mu \mathrm{M})$, or vehicle for $24 \mathrm{~h}$. The cells were then fixed with $4 \%$ paraformaldehyde in PBS for 15 min, permeabilized in PBS containing 0.1\% Triton X-100 for 15 min and then rinsed with PBS. The fixed cells were incubated with anti-F-actin antibody conjugated with Alexa Fluor 594 phalloidin (Molecular Probes, Eugene, OR, USA) for $16 \mathrm{~h}$ at $4^{\circ} \mathrm{C}$ and rinsed with PBS. The nucleus was stained by incubation with Syto Green Fluorescent Nucleic Acid Stains (24 dye; Molecular Probes). A fluorescent microscope (Axiovert 200M, Carl Zwiss, Tokyo, Japan) was used to visualize the structure of F-actin.

Cell survival assay using $\mathrm{CuSO}_{4}$. SCC cells were treated with $\mathrm{CuSO}_{4}$ and cell viability was measured 24 and $48 \mathrm{~h}$ after the treatment using Countess Automated Cell Counter (Invitrogen, Eugene, OR, USA). The inhibition of cell growth was compared to vehicle-treated control.

RNA interference approach. SAS was trypsinized and resuspended in DMEM without FBS, and the cells were separated approximately $1 \times 10^{5}$ cells for each dish. The AQP5-specific siRNA (Stealth siRNA) was purchased from Invitrogen Japan Inc. (Tokyo, Japan). The sequence of the sense strand of AQP5-siRNA is 5'-CGGUGGUCAUGAAUCGGUUtt-3', and antisense is 5'-AACCGAUUCAUGACCACCGca-3', respectively. The sequence of the sense strand of AQP3-siRNA is 5'-GGGUCGUCACUCCUUUAAUtt-3', and antisense is 5'-AUUAAAGGAGUGACGACCCtt-3', respectively. For the transfection, AQP5-siRNA (40 nM) or negative control (40 nM Stealth RNAi Negative Control Duplex, Invitrogen Japan Inc.) solution was added to DMEM medium containing Lipofectamine RNAiMAX (Invitrogen Japan Inc.) and allowed to incubate for $20 \mathrm{~min}$ at room temperature to create the transfection mixture. The transfection mixture was then added to the cells at the indicated final concentration of siRNA. Twenty-four hours after the transfection, the medium was changed to DMEM containing $10 \%$ FBS. Then, viable cell number was measured $48 \mathrm{~h}$ after the medium change using Countess Automated Cell Counter. The cell growth was expressed as the percentage to that of vehicle control.

Adhesion assay. SAS was placed onto cell culture plates with DMEM without FBS. After the cells were completely attached, treatment with AQP5-siRNA (40 nM) was performed for $24 \mathrm{~h}$. The cells were then cultured for $24 \mathrm{~h}$ after the culture medium was changed to DMEM with FBS. Floating cells were observed, and the number of floating dead cells was counted.

Western blot analysis. Adherent or suspended cells were washed in PBS, and cell extracts were prepared by lysing cells in lysis buffer. The proteins were separated by electrophoresis using 10\% SDS-PAGE, and transferred to nitrocellulose membrane (Millipore, Bedford, MA, USA). Detection of proteins were performed by each polyclonal antibody and visualized by using the ECL detection kit (Amersham, London, UK) following the manufacturer's suggested procedure.

Combination of AQP5-siRNA and AQP3-siRNA. Combined treatment of AQP5-siRNA and AQP3-siRNA was performed. Briefly, after the low concentration of AQP5-siRNA (20 nM) and AQP3-siRNA $(20 \mathrm{nM})$ treatment for $24 \mathrm{~h}$, the medium was changed to DMEM containing $10 \%$ FBS. Then, viable cell number was measured $48 \mathrm{~h}$ after the medium change using Countess Automated Cell Counter.

Statistical analysis. Results are expressed as mean \pm SEM or \pm SD. Statistical comparisons were made using the Student's t-test or Scheffe's method after analysis of variances (ANOVA). The results were considered significantly different at $\mathrm{P}<0.05$. 
A

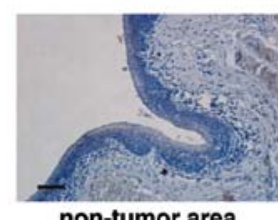

non-tumor area

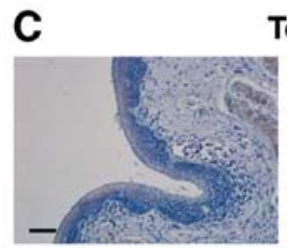

non-tumor area
Tongue tissues (AQP5)

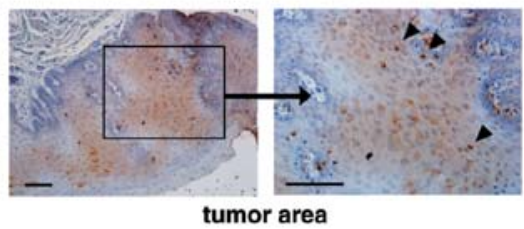

Tongue tissues (AQP3)

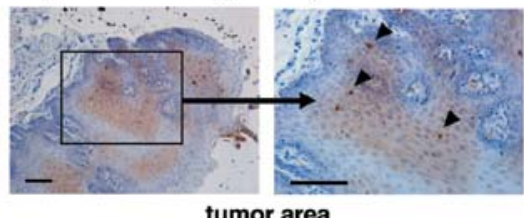

E

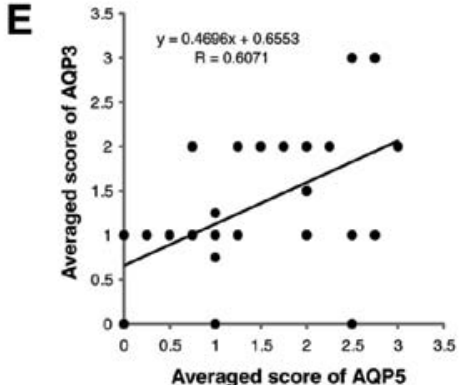

B $A Q P 5$
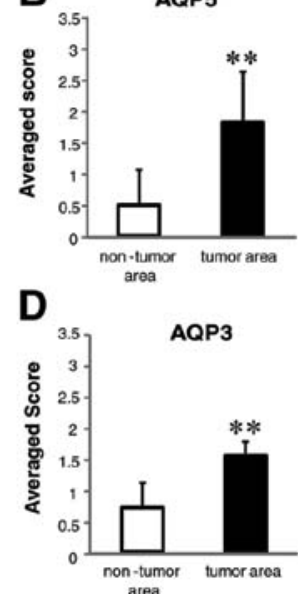

Figure 1. Expression of AQP5 or AQP3 on tumor cells in tongue tissues. (A) AQP5 expression on tumor area (middle and right panel) of tongue squamous cell carcinoma and non-tumor area (left panel) in the same tissue section (case 13 in Table I) by immunohistochemical observations. The brown color represents positive staining of AQP5 with blue counterstaining. Scale bar represents $100 \mu \mathrm{m}$. (B) Comparison of the expression of AQP5 between tumor area and non-tumor area. Averaged score of AQP5 expression is expressed from the data in Table I. Each column represents mean $\pm \mathrm{SEM}$ from $22-24$ cases. ${ }^{* *} \mathrm{P}<0.01$. (C) AQP3 expression on tumor area (middle and right panel) of tongue squamous cell carcinoma and non-tumor area (left panel) in the same tissue section (case 13 in Table I) by immunohistochemical observations. The brown color represents positive staining of AQP3 with blue counterstaining. Scale bar, $100 \mu \mathrm{m}$. (D) Comparison of the expression of AQP3 between tumor area and non-tumor area. Averaged score of AQP3 expression is expressed based on the data in Table I. Each column represents mean \pm SEM from 24 cases. ${ }^{* *} \mathrm{P}<0.01$. (E) Correlation between AQP5 and AQP3 expression. Each point represents one individual.

\section{Results}

Tongue SCCs in tumor tissues and cultured SCC cell lines express both AQP5 and AQP3. SCC tissues of the tongue were stained using anti-AQP5 or anti-AQP3 specific antibody. Positive staining of AQP5 was observed in tumor area (Fig. 1A, middle and right panel). In contrast, weak staining was observed in non-tumor area in the same tissue section (Fig. 1A, left panel). Similar staining pattern was also observed in other cases (Table I). Statistically significance of the AQP5 expression between tumor and non-tumor area was observed (Fig. 1B). In addition, positive staining of AQP3 in tumor area, but not in non-tumor area, was also observed in the same tissue section (Fig. 1C). Statistically significance of the AQP3 expression between tumor and non-tumor area was also observed (Fig. 1D and Table I). The expression patterns were similar between AQP5 and AQP3. Good correlation between AQP5 and AQP3 expression was observed (Fig. 1E).

Expression of AQP5 and AQP3 on malignant salivary gland tumor tissues. There are no reports on the expression of AQP5 or AQP3 on malignant salivary gland tumors. ACC tissues were stained using anti-AQP5 and anti-AQP3 specific antibody, respectively. Positive staining of AQP5 was observed in non-tumor area (Fig. 2A, left panel). The positive stainings of
AQP5 was observed in normal glands. In contrast, only slight staining of AQP5 was observed in tumor area in the same tissue section (Fig. 2A, middle and right panel). Similar staining pattern was also observed in other tumor tissue sections (Table II). Statistical significance of the AQP5 expression between tumor and non-tumor area was observed (Fig. 2B). These results are the opposite to the significant expression of AQP5 was mainly observed in tumor area, not in non-tumor area, of SCC tissues. In addition, positive staining of AQP3 in non-tumor area, but not in tumor area, was also observed in the same tissue section (Fig. 2C). Statistical significance of the AQP3 expression between tumor and non-tumor areas was also observed (Fig. 2D and Table II). These results are similar to that of AQP5. Our observations may indicate that the roles of AQP5 and AQP3 are different between SCCs and salivary gland tumors.

Pan-AQP inhibitor suppresses cell growth and changes cell shape of tongue SCC. Western blot analysis showed the expressions of AQP5 and AQP3 in oral SCC cell lines (SAS, SCCKN and CA9-22) (Fig. 3A). In contrast, both AQP5 and AQP3 expression in fibroblasts was much weaker than that in SCCs.

According to the data on AQP5 and AQP3 expression in SCCs, we hypothesized that both AQP functions might play an important role on the cell growth of SCCs. To investigate 
Table I. Profile of tongue squamous cell carcinoma patients, the histological diagnosis and expression of AQP3 and AQP5 in tissue sections.

\begin{tabular}{|c|c|c|c|c|c|c|}
\hline \multirow[b]{2}{*}{ Case } & \multirow[b]{2}{*}{$\begin{array}{c}\text { Age/ } \\
\text { gender }\end{array}$} & \multirow[b]{2}{*}{ Differentiation } & \multicolumn{2}{|c|}{ AQP3 expression } & \multicolumn{2}{|c|}{ AQP5 expression } \\
\hline & & & Tumor area & $\begin{array}{c}\text { Non-tumor } \\
\text { area }\end{array}$ & Tumor area & $\begin{array}{c}\text { Non-tumor } \\
\text { area }\end{array}$ \\
\hline 1 & $71 / \mathrm{F}$ & Well differentiated SCC & +++ & + & $++\sim+++$ & $-\sim+$ \\
\hline 2 & $57 / \mathrm{M}$ & Well differentiated SCC & ++ & + & $+\sim++$ & - \\
\hline 3 & 69/M & Well differentiated SCC & ++ & + & ++ & + \\
\hline 4 & 64/M & Well differentiated SCC & ++ & + & $++\sim+++$ & $-\sim+$ \\
\hline 5 & $46 / \mathrm{F}$ & Well differentiated SCC & ++ & + & +++ & + \\
\hline 6 & 48/M & Well differentiated SCC & ++ & + & $+\sim++$ & - \\
\hline 7 & $72 / \mathrm{M}$ & Well differentiated SCC & + & + & $+\sim++$ & - \\
\hline 8 & 79/M & Well differentiated SCC & - & + & $++\sim+++$ & + \\
\hline 9 & $34 / \mathrm{M}$ & Well differentiated SCC & $+\sim++$ & - & + & - \\
\hline 10 & $68 / \mathrm{M}$ & Moderately differentiated SCC & ++ & + & $+\sim++$ & - \\
\hline 11 & $64 / \mathrm{M}$ & Moderately differentiated SCC & +++ & + & $++\sim+++$ & + \\
\hline 12 & $58 / \mathrm{F}$ & Moderately differentiated SCC & ++ & + & +++ & N/A \\
\hline 13 & $92 / \mathrm{F}$ & Moderately differentiated SCC & $+\sim++$ & - & ++ & - \\
\hline 14 & $86 / \mathrm{F}$ & Moderately differentiated SCC & ++ & + & $+\sim++$ & $-\sim+$ \\
\hline 15 & $57 / \mathrm{M}$ & Moderately differentiated SCC & ++ & + & +++ & ++ \\
\hline 16 & $62 / \mathrm{M}$ & Moderately differentiated SCC & + & + & $++\sim+++$ & + \\
\hline 17 & $52 / \mathrm{F}$ & Poorly-moderately differentiated SCC & + & - & + & - \\
\hline 18 & 38/M & Poorly differentiated SCC & ++ & + & $+\sim++$ & N/A \\
\hline 19 & $51 / \mathrm{F}$ & Poorly differentiated SCC & + & - & + & - \\
\hline 20 & $67 / \mathrm{M}$ & Poorly differentiated SCC & + & + & $-\sim+$ & - \\
\hline 21 & $66 / \mathrm{M}$ & Poorly differentiated SCC & + & - & $++\sim+++$ & + \\
\hline 22 & $65 / \mathrm{M}$ & Poorly differentiated SCC & ++ & + & $-\sim+$ & - \\
\hline 23 & 33/M & Poorly differentiated SCC & $-\sim+$ & - & + & - \\
\hline 24 & 30/M & Poorly differentiated SCC & ++ & + & $+\sim++$ & + \\
\hline
\end{tabular}

Expression of AQP3 or AQP5 by immunohistochemical staining in tumor and non-tumor area is scored and expressed as (-) to (+++). N/A, not applicable.

A

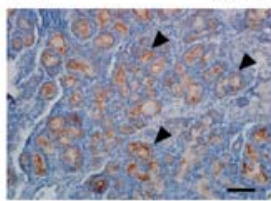

non-tumor area

C

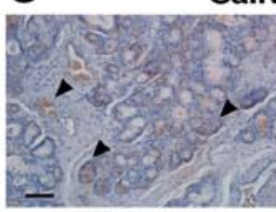

non-tumor area
Salivary gland tissue (AQP5)

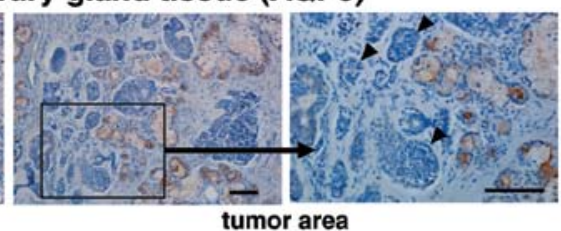

Salivary gland tissue (AQP3)

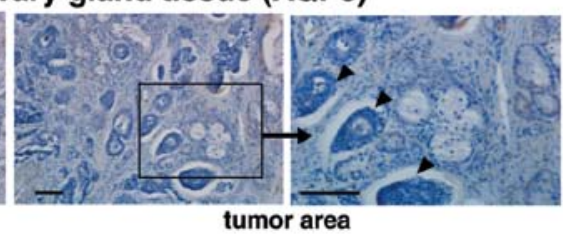

B
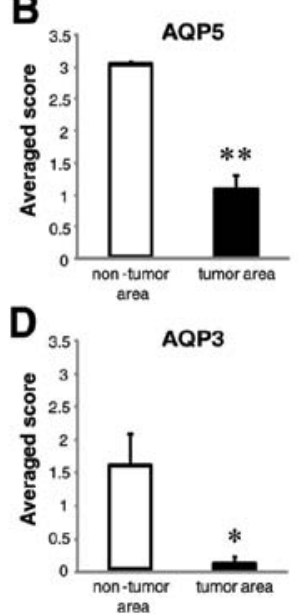

Figure 2. Expression of AQP5 and AQP3 on tumor cells in adenoid cystic carcinoma. (A) AQP5 expression on tumor area (middle and right panel) of salivary gland tumor (adenoid cystic carcinoma: ACC) and non-tumor area (left panel) in the same tissue section (case 2 in Table II) by immunohistochemical observations. The brown color represents positive staining of AQP5 with blue counterstaining. Scale bar, $100 \mu \mathrm{m}$. (B) Comparison of the expression of AQP5 between tumor area and non-tumor area. Averaged score of AQP5 expression is based on the data in Table II. Each column represents mean \pm SEM from $8-13$ cases. ${ }^{* *}<0.01$. (C) AQP3 expression on tumor area (middle and right panel) of salivary gland tumor (adenoid cystic carcinoma: ACC) and non-tumor area (left panel) in the same tissue section (case 2 in Table II) by immunohistochemical observations. The brown color represents positive staining of AQP3 with blue counterstaining. Scale bar, $100 \mu \mathrm{m}$. (D) Comparison of the expression of AQP3 between tumor area and non-tumor area. Averaged score of AQP3 expression is based on the data in Table II. Each column represents mean \pm SEM from 6-13 cases. ${ }^{*} \mathrm{P}<0.05$. 
Table II. Profile of malignant salivary gland tumor (adenoid cystic carcinoma) patients, the histological diagnosis and expression of AQP3 and AQP5 in tissue sections.

\begin{tabular}{|c|c|c|c|c|c|c|c|}
\hline \multirow[b]{2}{*}{ Case } & \multirow[b]{2}{*}{$\begin{array}{c}\text { Age/ } \\
\text { gender }\end{array}$} & \multirow[b]{2}{*}{ Histological type } & \multirow[b]{2}{*}{$\begin{array}{l}\text { Location } \\
\text { (gland) }\end{array}$} & \multicolumn{2}{|c|}{ AQP3 expression } & \multicolumn{2}{|c|}{ AQP5 expression } \\
\hline & & & & Tumor area & $\begin{array}{c}\text { Non-tumor } \\
\text { area }\end{array}$ & Tumor area & $\begin{array}{c}\text { Non-tumor } \\
\text { area }\end{array}$ \\
\hline 1 & $35 / \mathrm{M}$ & Adenoid cystic carcinoma & Submandibular & - & N/A & + & N/A \\
\hline 2 & $37 / F$ & Adenoid cystic carcinoma & Palatal & - & ++ & - & +++ \\
\hline 3 & $64 / \mathrm{M}$ & Adenoid cystic carcinoma & Buccal & - & - & $-\sim+$ & +++ \\
\hline 4 & $29 / \mathrm{F}$ & Adenoid cystic carcinoma & Parotid & - & N/A & $-\sim+$ & N/A \\
\hline 5 & $72 / \mathrm{F}$ & Adenoid cystic carcinoma & Buccal & - & N/A & $-\sim+$ & N/A \\
\hline 6 & $51 / \mathrm{F}$ & Adenoid cystic carcinoma & Floor of mouth & - & + & + & +++ \\
\hline 7 & $68 / \mathrm{F}$ & Adenoid cystic carcinoma & Floor of mouth & - & - & - & +++ \\
\hline 8 & $62 / \mathrm{M}$ & Adenoid cystic carcinoma & Palatal & + & +++ & +++ & +++ \\
\hline 9 & $62 / \mathrm{M}$ & Adenoid cystic carcinoma & Palatal & - & N/A & + & N/A \\
\hline 10 & $79 / \mathrm{F}$ & Adenoid cystic carcinoma & Floor of mouth & - & ++ & $-\sim+$ & +++ \\
\hline 11 & $79 / \mathrm{F}$ & Adenoid cystic carcinoma & Floor of mouth & - & N/A & + & N/A \\
\hline 12 & $59 / \mathrm{M}$ & Adenoid cystic carcinoma & Buccal & - & N/A & ++ & +++ \\
\hline 13 & $45 / F$ & Adenoid cystic carcinoma & Palatal & - & N/A & $+\sim++$ & +++ \\
\hline
\end{tabular}

Expression of AQP3 or AQP5 by immunohistochemical staining in tumor and non-tumor area is scored and expressed as (-) to (+++). N/A, not applicable.

the hypothesis, we used pan-AQP inhibitor, $\mathrm{CuSO}_{4}$. As shown in Fig. $3 \mathrm{~B}$ and $\mathrm{C}, \mathrm{CuSO}_{4}$ suppressed the cell growth of the tongue SCC cell line SAS. The suppression by the inhibitor was concentration-dependent (Fig. 3C). In contrast, $\mathrm{CuSO}_{4}$ did not suppress the cell growth of fibroblasts (Fig. 3E and F). These results indicate that $\mathrm{AQP}$ is required for the growth or survival of SCCs, but not of fibroblasts.

For visualization of the cell skeleton, we carried out fluorescent detection of F-actin by fluorescent microscopy. In the control group of SAS, the actin filaments were clearly aligned on the periphery in the cytoplasm (Fig. 3D, upper panel). When SAS cells were treated with $\mathrm{CuSO}_{4}(100 \mu \mathrm{M})$ for $24 \mathrm{~h}$, they became rounded with irregular disrupted actin alignment (Fig. 3D, lower panel). These results suggest that disruption of the cell skeletal structures by $\mathrm{CuSO}_{4}$ may consequently inhibit the cell's ability to keep the cell adhesion in SCCs. In contrast, the irregular shape was not seen in fibroblasts treated with $\mathrm{CuSO}_{4}$ (Fig. 3G). These results indicate that the inhibition of highly-expressed AQP functions may lead to effects such as cell growth inhibition and cytoskeletal disorders, in contrast to no effects on low expression cells.

AQP5-siRNA suppresses cell growth of tongue SCC. It is useful to down-regulate the highly expressed AQPs for clarifying the roles and their mechanisms of AQPs on SCCs. We have previously reported that knockdown of AQP3 by specific siRNA caused suppression of SCC growth (17). However, the role of AQP5 has not been investigated on the growth of SCC cell lines. In order to clarify the exact function of AQP5 on the growth of SCCs, we used small interfering RNA (siRNA) for AQP5. The inhibition of cell growth on SAS was clearly observed when AQP5 was knocked down by the treatment with AQP5-siRNA (Fig. 4A and B). These results clearly indi- cate that not only the AQP3 but also AQP5 is required for the growth or survival of SCCs.

The treated cells started floating after the start of the treatment, and some cells were floating and dead after $24 \mathrm{~h}$ (Fig. 4C and D). The percentage of dead cells within the floating cells was only $5 \%$ or less at $6 \mathrm{~h}$. In contrast, the dead cells within the floating cells were increased at 12 or $24 \mathrm{~h}$ (Fig. 4D). Concequently, the total number of cells was decreased (Fig. 4B). These results suggest that suppression of AQP5 initially caused the inhibition of cell adhesion and resulted in the inhibition of cell growth.

Investigation of potential mechanisms. We next investigated the mechanisms of inhibition of cell growth induced by the suppression of AQP5. Integrin $\alpha 5, \beta 1$ and FAK expressions were suppressed by the specific siRNA for AQP5 (Fig. 4E). The suppression of Erk, one of the important members of MAPK pathway, was also observed when SAS was treated with AQP5-siRNA (Fig. 4E). These results suggest that the cell growth suppression of SCCs by the knockdown of AQP5 is initially mediated through the inhibiton of integrin expression, and then through the inhibition of MAPK signalling pathway.

Combination of AQP5-siRNA and AQP3-siRNA. Although we observed that AQP5 or AQP3, respectively, played an important role in SCC growth, combined suppression both of AQP5 and AQP3 is still clear. Combined treatment of AQP5-siRNA (20 nM) and AQP3-siRNA (20 nM), the half concentration of single siRNA treatment (40 $\mathrm{nM})$, inhibited the cell growth of SAS similarly in comparison to that in each single high concentrate treatment (Fig. 4F). These results indicate that combination of AQP5-siRNA and AQP3-siRNA shows additive effect, but not synergetic, on the suppression of SCC growth. 

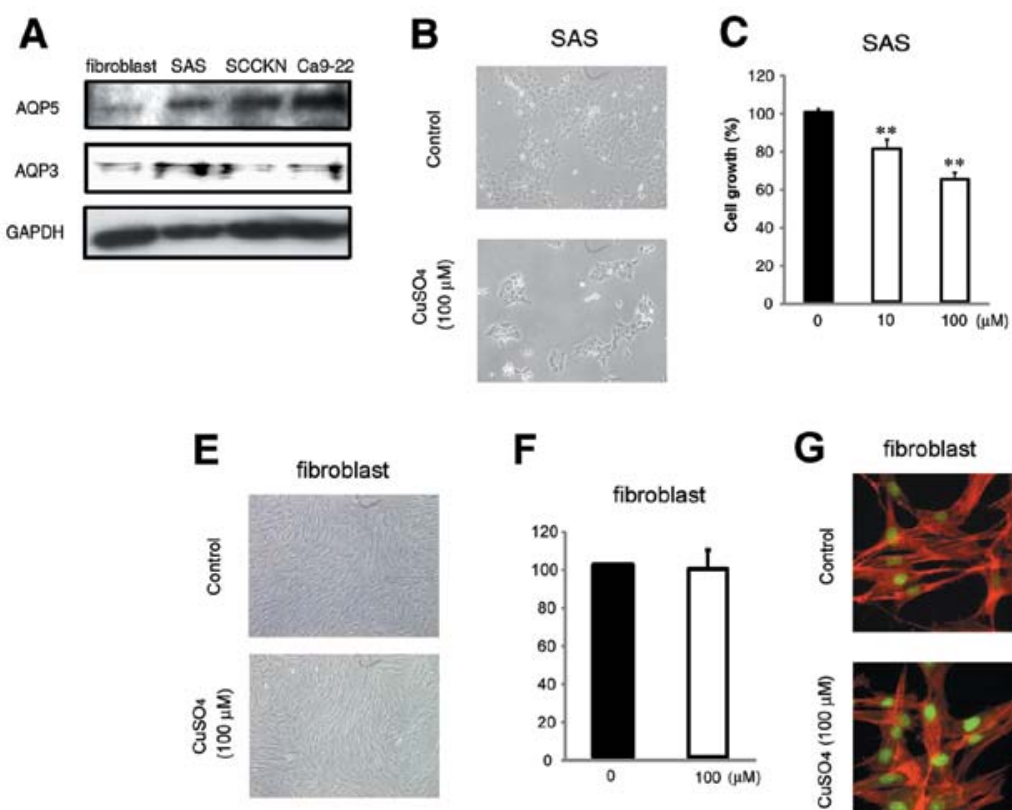

$\mathbf{F}$

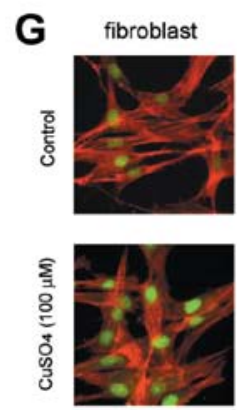

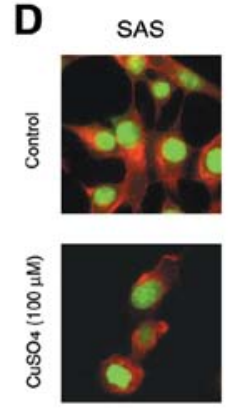

Figure 3. Expression of AQP5 in cultured tumor cell lines and effects of pan-AQP antagonist on SCC or fibroblast growth. (A) A representative western blot analysis showing AQP5 and AQP3 expressions in fibroblast and various oral SCC cell lines. GAPDH is standard for equivalent application. (B and C) Effect of $\mathrm{AQP}$ inhibitor, $\mathrm{CuSO}_{4}$ on cell growth of SAS. (B) Typical photos and (C) cell growth rate. Forty-eight hours after the start of treatment, viable cell number was counted and cell growth rate was expressed. Each column represents the percentage of cell growth (mean \pm SEM from 15 independent experiments) compared to vehicle control (Black column, PBS). Error bars represent standard errors. ${ }^{* *} \mathrm{P}<0.01$ vs. vehicle control. (D) Effect of AQP inhibitor on SAS cell shape change. F-actin in SAS cells treated with $\mathrm{CuSO}_{4}(100 \mu \mathrm{M})$ for $24 \mathrm{~h}$ was analyzed by fluorescent microscopy. Red color represents F-actin, and green color represents the nucleus. (E and F) Effect of AQP inhibitor, $\mathrm{CuSO}_{4}$ on cell growth of fibroblasts. (E) Typical photos and (F) cell growth rate. Forty-eight hours after the start of treatment, viable cell number was counted and cell growth rate was expressed. Right column represents the percentage of cell growth (mean \pm SEM from 8 independent experiments) compared to vehicle control (Black column, PBS). Error bars represent standard deviation. (G) Effect of AQP inhibitor on cell shape change of fibroblasts. F-actin in fibroblasts treated with $\mathrm{CuSO}_{4}(100 \mu \mathrm{M})$ for $24 \mathrm{~h}$ was analyzed by fluorescent microscopy. Red color represents F-actin and green color represents the nucleus.

\section{Discussion}

There have been several reports on the expression of AQP5 in various human tumors such as gastric, pulmonary, ovarian, pancreatic and colorectal cancer $(9,13-16)$. Therefore, it is considered that a relationship exists between AQP5 expression and tumor cell growth. However, there are few reports on the investigation of the expression and exact role of AQP5 on human SCC and ACC tissues.

In the present study, using immunohistochemistry, we demonstrated significantly higher levels of expression of AQP5 protein in human SCCs. Expression of AQP5 in tumor tissues was much higher than that in non-tumor areas in the same tissue samples. In addition, the expression of AQP3 was also overexpressed in the same tumor tissues and the positive correlation of the expression both of AQP5 and AQP3 was observed. These results indicate that both AQP5 and AQP3 are necessary for the proliferation or cell survival in oral SCCs.

Furthermore, we showed that the suppression of AQP5 protein or inhibition of function caused the inhibition of growth of SCC cell lines. In fact, in our experimental conditions, both the AQP inhibitor and AQP5-siRNA strongly inhibited the cell adhesion and growth of SCCs, but not of fibroblasts. These results suggest the important role of AQP5 in SCC adhesion and survival, but not in normal cells.

Contrary to the results of SCCs, we found that the expression of AQP5 on tumor area of ACC was down- regulated in comparison to that on non-tumor area in the same tissue section. Originally, AQP5 is localized mainly in the apical membrane, including intercellular secretory canaliculi of secretory cells in the minor salivary glands, pyloric glands, and duodenal glands and plays an important role in water transfer in these glands $(22,23)$. On the way to dedifferentiation and cancer development, AQP5 might be down-regulated in ACC.

The expression of AQP5 and AQP3 was observed both in human SCC tissues and SCC cell lines, but only slightly in non-tumor tissue and fibroblasts, indicating the important role of AQP5 and AQP3 in SCCs. We, therefore, used fibroblasts in order to investigate whether the inhibition of cell proliferation and change of cell structure occur or not because the expression both of AQP5 and AQP3 was quite low. When fibroblasts were treated with $\mathrm{CuSO}_{4}$, there was no difference on the cell growth and cytoskeletal structure compared with those of the control. These results indicate that the inhibition of low-expressed AQP functions may not show the obvious effects such as cell growth inhibition or cytoskeletal disorders.

The cell adhesion and growth were inhibited by the suppression of AQP5. Visible differences were not observed before $6 \mathrm{~h}$ after the treatment. However, the cells started floating $12 \mathrm{~h}$ after the start of the treatment, and some cells were floating and dead at 12-24 h. These results might suggest that the suppression of AQP5 protein or function causes the inhibition of cell adhesion, resulting in the inhibition of cell 
A

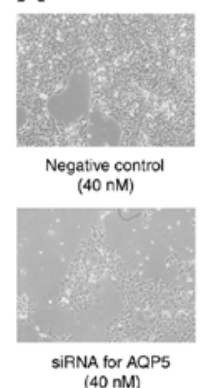

B

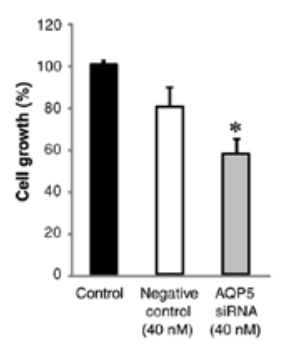

E

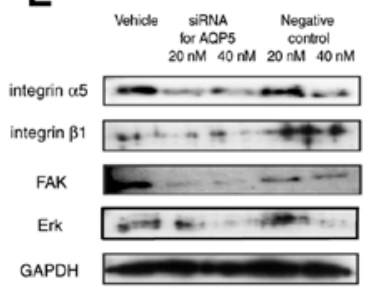

C

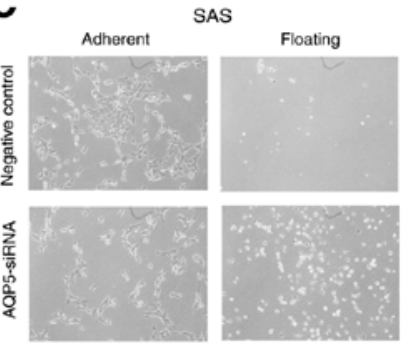

D

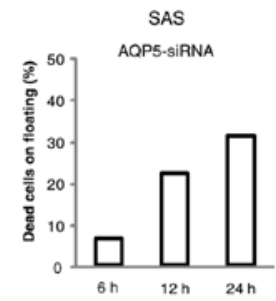

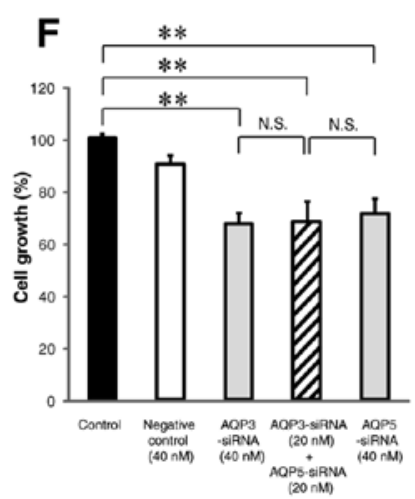

Figure 4. Effects of AQP5 knockdown on the cell growth of SCC treated with AQP5-siRNA and combination of AQP5-siRNA and AQP3-siRNA. (A and B) Effects of AQP5-siRNA on cell growth of SAS. (A) Typical photos and (B) cell growth rate. Cells were transfected the siRNA ( $40 \mathrm{nM}$ ) for $24 \mathrm{~h}$ and cultured for additional $48 \mathrm{~h}$, followed by viable cell counting. Each value represents the percentage of cell growth compared with vehicle (non-siRNA) control from 8 independent experiments. Gray column represents the growth of cells transfected with AQP5-siRNA and white column represents that of cells transfected with negative control-siRNA. Error bars represent standard errors. "P $<0.05$ vs. negative control. (C) Typical photographs of SAS treated with AQP5-siRNA. Cells were treated with AQP5-siRNA for $24 \mathrm{~h}$, and then the floating cells and adhesive cells were separated. The upper columns are composed of adherent cells and the lower ones are floating cells. (D) Percentage of dead cells within the floating cells after treatment with AQP5-siRNA. The dead cells within the total floating cells at 6,12 and $24 \mathrm{~h}$ after the treatment with AQP5-siRNA were counted and expressed as a percentage. The data are the average of 4-8 independent treatment. (E) Involvement of MAPK pathway on AQP signaling of SCC growth. Western blot analysis for expression of integrin $\alpha 5$, integrin $\beta 1, \mathrm{FAK}$ and Erk by the treatment with AQP5-siRNA on SAS. Cells were treated with AQP5-siRNA ( 20 and $40 \mathrm{nM}$ ), negative control siRNA and vehicle (Veh). Samples were collected for $24 \mathrm{~h}$ after the treatment. GAPDH was used to evaluate equivalent loading. (F) Combined treatment of low dose of AQP3-siRNA ( $20 \mathrm{nM})$ and AQP5-siRNA $(20 \mathrm{nM})$ was performed on SAS. Cells were treated with AQP3-siRNA plus AQP5-siRNA (20 nM each), AQP3-siRNA (40 nM), AQP5-siRNA (40 nM), negative control (40 nM) or vehicle for $24 \mathrm{~h}$, then the medium was changed for $48 \mathrm{~h}$. Each value represents the percentage of cell growth compared with vehicle (non-siRNA) control from 6-11 independent experiments. ${ }^{* *} \mathrm{P}<0.01$.

\section{A normal squamous cell tumor squamous cell}

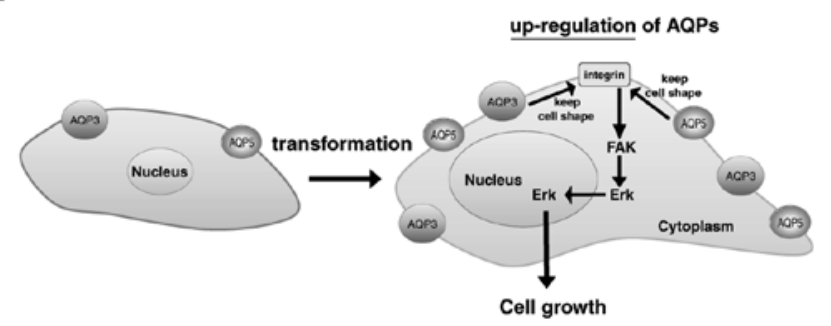

B

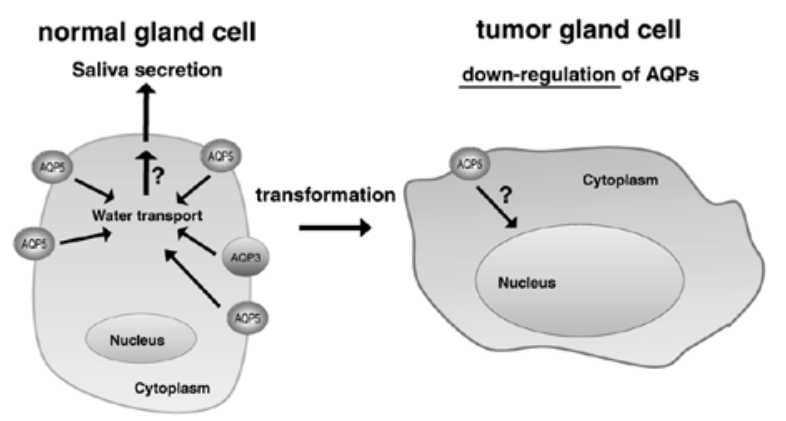

Figure 5. Schematic illustration of possible functions of AQP5 and AQP3 in SCC and ACC. (A) Alterations of AQP5 and AQP3 expressions during tumorigenesis of squamous cells. The expression of AQP3 and AQP5 are low in normal squamous cells (left pannel), but high in tumor squamous cells (right pannel). Arrow indcates the cell growth signaling through the AQP3 and AQP5 pathways. (B) Alterations of AQP5 and AQP3 expressions during tumorigenesis of gland cells. The expression of AQP3 and AQP5 are high in normal gland cells (left pannel), but low in tumor gland cells (right pannel). 
growth, namely anoikis (24). Anoikis, apoptosis resulting from loss of cell-matrix interaction, was first documented in normal epithelial cells and endothelial cells, and it helps maintain a dynamic balance between cell turnover and survival (24). Malignant cells are able to resist apoptosis to varying degrees, and this property has been proposed to contribute to tumorigenesis and metastasis $(24,25)$. It is well known that apoptosis and inhibition of cell growth in SCC cell lines are induced by anoikis, apoptosis resulting from loss of cell-matrix interaction, and FAK is reported to be involved in anoikis $(19,21,25)$. FAK is a $125 \mathrm{kDa}$ non-receptor tyrosine kinase and an important regulator of cell survival, invasion, migration, and cell cycle progression $(24,26,27)$. FAK is functionally important in transducing intracellular messages that are associated with growth factor signalling (22). Our results indicate that the mechanisms of the inhibition of cell growth by AQP5-siRNA are, in part, due to the inhibition of FAK-MAPK pathway in SCCs.

The activation of FAK signalling pathway is required for cell-extracellular matrix adhesion which is inhibited by decrease in integrin $\alpha 5$ and $\beta 1$ expression. Integrin $\alpha 5$ and $\beta 1$ are well known as the counter receptor for fibronectin $(28,29)$. Under the present experimental conditions, we confirmed that the integrin $\alpha 5$ and $\beta 1$ expressions were suppressed by the treatment with AQP5-siRNA, indicating the inhibition of cell-extracellular matrix adhesion. The cell-extracellular matrix adhesion is required for the continuous activation of FAK signalling pathway. Therefore, the decrease in integrin $\alpha 5$ and $\beta 1$ expressions by the suppression of AQP5 might be one of the potential mechanisms to inhibit the cell-extracellular matrix adhesion and resulting inhibition of FAK-MAPK signalling pathways (Fig. 5A). We have already reported the critical role of AQP3 in SCCs (17) and the signalling goes through the FAK-MAPK pathway via integrins. We, therefore, also explained that the AQP5 signalling may go through the FAK-MAPK pathway in this study. Further investigations might be necessary to clarify the detailed mechanisms.

Combined treatment of AQP5-siRNA (20 nM) and AQP3-siRNA (20 $\mathrm{nM})$ inhibited the cell growth of SAS similarly in comparison to that in each single high concentration $(40 \mathrm{nM})$ treatment. These are pharmacologically addditive effects. Because of the same pathway at the point of cell proliferation between AQP5 and AQP3, combination of AQP5-siRNA and AQP3-siRNA showed only additive effect, but not synergetic, on the suppression of SCC growth.

In SCCs, the expression of AQP5 is low in normal epithelial tissue originally, but its expression becomes high when cancer develops. The purpose of these changes may be, in part, to activate the MAPK pathway to acceralate the proliferation and to maintain the cell cytoskeleton.

In summary, we showed the overexpression of AQP5 and AQP3 in tumor cells of human tongue SCC and cultured SCC cell lines, and suggest a potentially important role for AQP5 and AQP3 on the cell growth of human SCCs. In contrast, AQP5 and AQP3 were down-regulated in tumor cells of human oral ACC. Therefore, the control of AQP expression and function might be a useful target in the therapy for oral SCC.

\section{Acknowledgements}

This study was supported in part by grants (21592357 to K.W., 20390471 to Y.K. and 21390535 to M.K.) from the Japanese Society for the Promotion of Science.

\section{References}

1. Goepfert H: Squamous cell carcinoma of the head and neck: past progress and future promise. CA Cancer J Clin 48: 195-198, 1998.

2. Okura M, Hiranuma T, Adachi T, et al: Induction chemotherapy is associated with an increase in the incidence of locoregional recurrence in patients with carcinoma of the oral cavity: results from a single institution. Cancer 82: 804-815, 1998.

3. Prince S and Bailey BM: Squamous carcinoma of the tongue: review. Br J Oral Maxillofac Surg 37: 164-174, 1999.

4. King LS and Agre P: Pathophysiology of the aquaporin water channels. Annu Rev Physiol 58: 619-648, 1996.

5. Verkman AS: More than just water channels: unexpected cellular roles of aquaporins. J Cell Sci 118: 3225-3232, 2005.

6. Kreda SM, Gynn MC, Fenstermacher DA, Boucher RC and Gabriel SE: Expression and localization of epithelial aquaporins in te adult human lung. AM J Respir Cell Mol Biol 24: 224-234, 2001.

7. Krane CM, Melvin JE, Nguyen HV, et al: Salivary acinar cells from aquaporin 5-deficient mice have decreased membrane water permeability and alterd cell volume regulation. J Biol Chem 276: 23413-23420, 2001.

8. Gresz V, Kwon TH, Gong H, et al: Immunolocalization of AQP-5 in rat parotid and submandibular salivary glands after stimulation or inhibition of secretion in vivo. Am J Physiol Gastrointest Liver Physiol 287: G151-G161, 2004.

9. Burghardt B,Elkaer ML, Kwon TH, et al: Distribution of aquaporin water channels AQP1 and AQP5 in the ductal system of the human pancreas. Gut 52: 1008-1016, 2003.

10. Verkman AS and Mitra AK: Structure and function of aquaporin water channel. Am J Physiol Renal Physiol 278: F13-28, 2000.

11. Fujiyoshi Y, Mitsuoka K, de Groot BL, et al: Structure and function of water channels. Curr Opin Struct Biol 12: 509-515, 2002.

12. Nico B and Ribatti D: Aquaporins in tumor growth and angiogenesis. Cancer Lett 294: 135-138, 2010.

13. Watanabe T, Fujii T, Oya T, et al: Involvement of aquaporin-5 in diffferentiation of human gastric cancer cells. J Physiol Sci 59: 113-122, 2009.

14. Zhang Z, Chen Z, Song Y, Zhang P, Hu J and Bai C: Expression of aquaporin 5 increases proliferation and metastasis potential of lung cancer. J Pathol 221: 210-220, 2010.

15. Yang JH, Shi YF, Cheng Q and Deng L: Expression and localization of aquaporin-5 in the epithelial ovarian tumors. Gynecol Oncol 100: 294-299, 2006.

16. Kang SK, Chae YK, Woo J, et al: Role of human aquaporin-5 in colorectal carcinogenesis. Am J Pathol 173: 518-525, 2008.

17. Kusayama M, Wada K, Nagata M, et al: Critical role of aquaporin 3 on growth of human esophageal and oral squamous cell carcinoma. Cancer Sci 102: 1128-1136, 2011.

18. Nagata M, Wada K, Nakajima A, et al: Role of myeloid cell leukemia-1 on cell growth of squamous cell carcinoma. J Pharmacol Sci 110: 344-353, 2009.

19. Masuda T, Wada K, Nakajima A, et al: Critical role of peroxisome proliferator-activated receptor gamma on anoikis and invasion of squamous cell carcinomas. Clin Cancer Res 11: 4012-4021, 2005.

20. Takahashi H, Fujita K, Fujisawa T, et al: Inhibition of peroxisome proliferator-activated receptor gamma activity in esophageal carcinoma cells results in a drastic decrease of invasive properties. Cancer Sci 97: 854-860, 2006.

21. Ishida H, Wada K, Masuda T, et al: Critical role of estrogen receptor on anoikis and invasion of squamous cell carcinoma. Cancer Sci 98: 636-643, 2007.

22. Matsuzaki T, Tajika Y, Suzuki T, Aoki T, Hagiwara H and Takata K: Immunolocalization of the water channel, aquaporin-5 (AQP5), in the rat digestive system. Arch Histol Cytol 66: 307-315, 2003.

23. Delporte $\mathrm{C}$ and Steinfeld S: Distribution and roles of aquaporins in salivary glands. Biochim Biophys Acta 1758: 1061-1070, 2006. 
24. Frish SM and Francis H: Distribution of epithelial cell-matrix interactions induces apoptosis. J Cell Biol 124: 619-626, 1994.

25. Yamada KM and Geiger B: Molecular interactions in cell adhesion complexes. Curr Opin Cell Biol 9: 76-85, 1997.

26. Chen HC, Appeddu PA, Parsons JT, Hildebrand JD, Schaller MD and Guan JL: Interaction of focal adhesion kinase with cytoskeletal protein talin. J Biol Chem 270: 16995-16999, 1995.

27. Owens LV, Xu L, Dent GA, et al: Focal adhesion kinase as a marker of invasive potential in differentiated human thyroid cancer. Ann Surg Oncol 3: 100-105, 1996.
28. Zhang Z, Vuori K, Reed JC and Ruoslahti E: The alpha 5 beta 1 integrin supports survival of cells on fibronectin and up-regulates Bcl-2 expression. Proc Natl Acad Sci USA 92: 6161-6165, 1995.

29. Sieg DJ, Hauck CR, Ilic D, et al: FAK integrates growth-factor and integrin signals to promote cell migration. Nat Cell Biol 2: $249-256,2000$ 